\title{
A comparison of the oxidative stress response in single-incision laparoscopic versus multi-trocar laparoscopic totally extraperitoneal inguinal hernia repair
}

\author{
Jacek T. Białecki ${ }^{1}$, Waldemar Myszka², Ewa Wysocka², Sebastian Sowier ${ }^{1}$, Przemysław Pyda ${ }^{3}$, Ryszard Antkowiak ${ }^{4}$, \\ Łukasz Antkowiak ${ }^{5}$, Anna Sowier ${ }^{1}$, Zbigniew Krasiński ${ }^{6}$ \\ ${ }^{1}$ Department of General Surgery, Franciszek Raszeja Memorial Hospital, Poznan, Poland \\ ${ }^{2}$ Department of Clinical Biochemistry and Laboratory Medicine, Chair of Chemistry and Clinical Biochemistry, Poznan University \\ of Medical Sciences, Poznan, Poland \\ ${ }^{3}$ Department and Clinic of General and Endocrine Surgery and Gastroenterological Oncology, Faculty of Medicine I, Poznan University \\ of Medical Sciences, Poznan, Poland \\ ${ }^{4}$ Department of General Surgery, Regional Health Centre, Lubin, Poland \\ ${ }^{5}$ Department of Histology and Embryology, Medical University of Silesia, Zabrze, Poland \\ ${ }^{6}$ Department of General and Vascular Surgery, Poznan University of Medical Sciences, Poznan, Poland
}

Videosurgery Miniinv 2020; 15 (4): 567-573 DOI: https://doi.org/10.5114/wiitm.2020.93202

\begin{abstract}
Introduction: Surgical treatment is always associated with tissue damage and the subsequent development of oxidative stress.

Aim: To compare the oxidative stress response in patients treated operatively for inguinal hernia with multi-trocar laparoscopic totally extraperitoneal technique (TEP) or single-incision laparoscopic totally extraperitoneal technique (TEP-SI).

Material and methods: A randomized group of 34 patients with one-sided inguinal hernia was enrolled in the study. Seventeen patients were treated with a standard TEP method (group 1) and the other 17 patients were treated with the TEP-SI technique (group 2). Thiobarbituric acid reactive substances (TBARS) and total antioxidant status (TAS) as the oxidative stress markers were measured before surgery (0), 1 day (1) and 4 days (2) after surgery.

Results: A decrease in TAS on the first day after surgery was observed in both groups. Sustained reduction on the fourth day after surgery was observed in group 1, whereas in group 2 an increase followed. A statistically significant difference was observed in TAS $(2: 0)$ ratio with a meaningful decrease in group 1. TBARS concentration was elevated 1 day after surgery in both groups. It remained at an elevated level on the fourth day after surgery in group 1 , while it decreased in group 2. The duration of surgery was higher in group 2 (mean: $57.5 \mathrm{~min}$ ) than in group 1 (mean: $50.0 \mathrm{~min})(p=0.0286)$.

Conclusions: Oxidative stress levels are lower in patients treated operatively by TEP-SI technique than by TEP. TEP-SI may be considered as a less invasive technique associated with less tissue injury.
\end{abstract}

Key words: minimally invasive surgery, oxidative stress, inguinal hernia repair, single-incision laparoscopic surgery. 


\section{Introduction}

Surgical treatment, regardless of the method used, always results in tissue damage and the subsequent metabolic response of the organism [1, 2]. There is no single and major parameter of measuring oxidative stress; thus many biomarkers can be used in different settings [3]. Numerous clinical trials have focused on the comparison of the organism's clinical and metabolic reactions following classic vs. laparoscopic surgery [4-8]. The results of those studies have proven that minimally invasive surgery results in less severe damage and is generally less harmful to the patient. This, in turn, positively correlates with healing, a lower proportion of postoperative complications and faster return to normal physical activity. The next step in the development of minimally invasive techniques was single-port laparoscopy, where an optical trocar and surgical instruments are introduced via the same port-trocar called a multiport. This enables only one incision to be used instead of three. Among the advantages of single-port laparoscopy better cosmesis, lower pain levels, and a smaller number of complications are usually mentioned [9-11]. Based on the current European Hernia Society guidelines, the methods of choice for the treatment of inguinal hernia are the Lichtenstein and totally extra peritoneal (TEP) repairs [12]. Lately, single incision technique (TEP-SI) is growing in popularity in hernia operations [13, 14]. A number of studies comparing single-incision laparoscopic totally extra-peritoneal inguinal hernia repair with standard TEP procedure have been published in recent years [15-18]. However, they did not prove a significant advantage of TEP-SI over TEP. Instead, TEP-SI is considered a more difficult technique due to the loss of triangulation and external and internal conflict $[19,20]$. In this paper we try to investigate whether TEP-SI has an advantage over the standard TEP procedure by analyzing the oxidative stress levels, which reflect the extent of the patient's surgical trauma.

\section{Aim}

The aim of the study was to compare the oxidative stress response in patients treated operatively for inguinal hernia with TEP or TEP-SI technique. In order to do that, thiobarbituric acid reactive substances (TBARS) and total antioxidant status (TAS) were measured and compared before and after surgery.

\section{Material and methods}

The study was performed in accordance with the Declaration of Helsinki for Human Research 1975. The study protocol was approved by the appropriate bioethics committee. The participants of the study gave informed consent to the study procedure. A randomized group of 34 patients with one-sided inguinal hernia (M1 or L1 according to the EHS classification, with the width $<1.5 \mathrm{~cm}$ ) were enrolled in the study. All the patients had a normal body mass index (BMI), did not suffer from chronic diseases, had not been using long-term medications and presented no signs of active infection during surgery. 17 patients were treated with a standard TEP method (group 1) and the other 17 patients were treated with the TEP-SI technique (group 2).

\section{Surgical approach - TEP-SI}

The procedure was performed under general anesthesia. First, the surgical site was disinfected. Next, a $25 \mathrm{~mm}$-long transverse incision was made to the side of the hernia, above the umbilicus and then Langenbeck hooks were used to retract the wound edges. As a result, the anterior surface of the rectus sheath of the rectus abdominis muscle was revealed. The rectus abdominis medial edge was revealed following the incision of the anterior rectus sheath. Having moved the muscle aside, the index finger was placed beneath it and then moved down across the muscle's posterior surface. This allowed the preperitoneal area to be dissected. The following steps involved introduction of a $10 \mathrm{~mm}$-trocar in the area, administration of carbon dioxide and insertion of a surgical camera. The camera was then set in a swinging motion in order to dissect the preperitoneal area towards ASIS to the pubic symphysis. Next, two dissectors were introduced below the umbilicus. Packing suture was used for skin suturing so that pneumoperitoneum was maintained between the surgical instrument and the trocar in the preperitoneal area (Photo 1).

The dissectors were used to continue dissection in the preperitoneal area. First, it was dissected below the pubic symphysis in the midline and then to the side, when the lower edge of Douglas' line was found. Next the hernia sac was dissected and then removed from the inguinal canal. With lateral inguinal hernia, spermatic cord elements were also dissected from the sac. The extent of dissection was 
the same in each type of hernia. The next stage involved inserting a $15 \times 13 \mathrm{~cm}$ propylene mesh. Then the desufflation procedure was performed using the camera to observe how the peritoneum spread on the mesh. It was not necessary to suture the mesh. A single absorbable suture was used to close the incision of the anterior rectus sheath of the rectus abdominis muscle. Then the skin incision was closed with an intra-dermal suture.

\section{Surgical approach - TEP}

The procedure was performed under general anesthesia. After the disinfection of the surgical site, a $13 \mathrm{~mm}$-long transverse incision was made to the side of the hernia, above the umbilicus. The operative technique was the same as in the TEP-SI approach up to the stage of pre-peritoneal area dissection. Instead of inserting two $5 \mathrm{~mm}$ tools next to the optical trocar, two additional incisions were made and the instruments were inserted. The first one was introduced through the $5 \mathrm{~mm}$ trocar (a $6 \mathrm{~mm}$ incision in the midline, around $3 \mathrm{~cm}$ below the incision for the optical trocar) and the second one was inserted through the second $6 \mathrm{~mm}$ incision, made close to the anterior superior iliac spine of the hernia side. The subsequent steps were the same as in the TEP-SI procedure.

\section{Blood sampling and biochemical analysis}

Ulnar venous blood samples were collected from all the participants three times (fasting, between 6:00 AM and 7:00 AM): before surgery (0), 1 day (1) and 4 days (2) after surgery. Baseline blood samples (0) were used for routine pre-operative laboratory assessment, including complete blood count, basic coagulation tests, C-reactive protein, glucose electrolyte levels and oxidative stress markers including TAS and TBARS measurements. Post-surgical blood samples (1 and 2) included assessment of TAS and TBARS only. Next, ratios expressing changes of these levels in time were calculated $(1: 0,2: 1 ; 2: 0)$. The quotient of approximately 1.00 for any TAS ratio or any TBARS ratio means that there were no changes of total antioxidant status or lipid peroxidation products in plasma between the time points indicated. We also proposed an index which evaluates changes in TAS concentrations in relation to changes in TBARS levels, i.e. TAS (1:0)/TBARS (1:0). TAS were measured spectrophotometrically (RANDOX Labora-

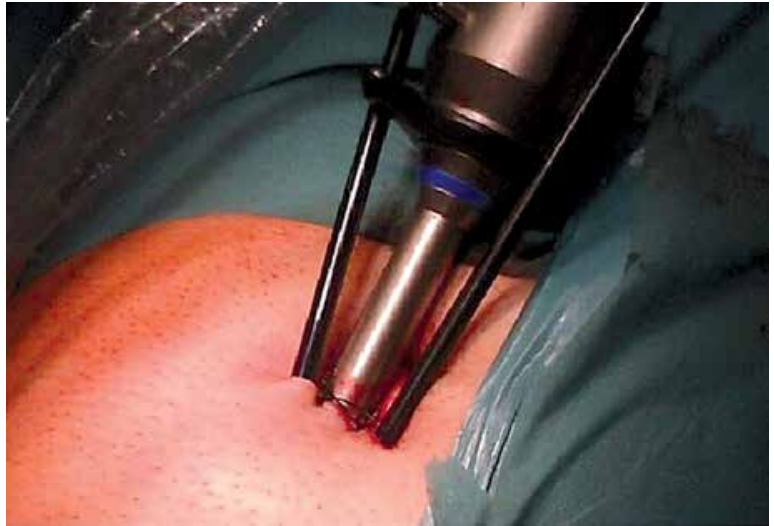

Photo 1. An optical trocar and surgical instruments introduced via the same $25-\mathrm{mm}$ incision

tories, Crumlin, Antrim, UK) using Statfax 1904Plus (Awareness Technology, Palm City, FL) and TBARS were determined according to the Okhawa method [21] (Sigma reagents, Germany) using Specord M40 (Carl-Zeiss, Germany). The intra- and inter-assay coefficients of variation were as follows: TAS - $1.6 \%$ and $3.7 \%$, TBARS $-1.9 \%$ and $3.8 \%$.

\section{Statistical analysis}

Statistical analysis was performed using Statistica software version 10.0. The normality of value distribution was checked using the Shapiro-Wilk test. Next, nonparametric analyses were used to compare two study groups (Mann-Whitney $U$ test) and to assess the changes of parameters during the time of observation (Friedmann ANOVA). A $p<0.05$ was chosen to indicate a significant difference. Data are presented as median and interquartile range (in parentheses).

\section{Results}

There were no statistically significant differences in age, preoperative (0 blood sample) WBC and CRP levels, TAS and TBARS concentrations between the two groups of patients. Preoperative data of the two study groups are presented in Table I.

The duration of surgery was higher in group 2 (mean: $57.5 \mathrm{~min}$ ) than in group 1 (mean: $50.0 \mathrm{~min}$ ), $p=0.0286$. The analysis of TAS concentrations at the three time points showed a decreased total antioxidant status on the first day after surgery (1 blood sample) in both groups. Sustained reduction on the fourth day after surgery (2 blood sample) was observed in group 1, whereas in group 2 an increase followed (Figures 1, 2). 
Jacek T. Białecki, Waldemar Myszka, Ewa Wysocka, Sebastian Sowier, Przemysław Pyda, Ryszard Antkowiak, Łukasz Antkowiak, Anna Sowier, Zbigniew Krasiński

Table I. Characteristics of the study groups

\begin{tabular}{|c|c|c|c|}
\hline Parameter & Group $1(n=17)$ & Group $2(n=17)$ & Significant difference \\
\hline Age [years] & $49.5(40.0-57.5)$ & $48.0(39.5-62.5)$ & NS \\
\hline CRP $[\mathrm{mg} / \mathrm{l}]$ & $1.49(0.83-4.19)$ & $1.43(0.76-4.33)$ & NS \\
\hline $\mathrm{WBC}\left[\times 10^{9} / 1\right]$ & $6.62(5.32-7.76)$ & $6.29(5.24-7.81)$ & NS \\
\hline $\mathrm{RBC}\left[\times 10^{12} / \mathrm{I}\right]$ & $4.91(4.63-5.21)$ & $4.97(4.27-5.14)$ & NS \\
\hline $\mathrm{HGB}[\mathrm{mmol} / \mathrm{l}]$ & $9.30(8.85-9.90)$ & $9.40(8.60-9.90)$ & NS \\
\hline HCT $[I / I]$ & $0.43(0.41-0.47)$ & $0.45(0.41-0.46)$ & NS \\
\hline $\mathrm{PLT}\left[\times 10^{9} / \mathrm{I}\right]$ & $213(193-245)$ & $205(191-257)$ & NS \\
\hline D-dimers [ $\mu \mathrm{g} / \mathrm{l}]$ & $368(184-502)$ & $357(196-437)$ & NS \\
\hline APTT ratio & $0.88(0.84-0.98)$ & $0.95(0.85-1.03)$ & NS \\
\hline INR & $1.03(0.97-1.09)$ & $1.03(0.98-1.04)$ & NS \\
\hline $\mathrm{Na}^{+}[\mathrm{mmol} / \mathrm{l}]$ & 140 (139-141) & 139 (138-142) & NS \\
\hline $\mathrm{K}^{+}[\mathrm{mmol} / \mathrm{l}]$ & $4.50(4.30-4.80)$ & $4.50(4.40-5.00)$ & NS \\
\hline $\mathrm{Cl}^{-}[\mathrm{mmol} / \mathrm{l}]$ & $105.0(102.5-107.5)$ & 104.0 (101.0-106.0) & NS \\
\hline Glucose [mg/dl] & $99.0(91.5-105.0)$ & 98.5 (91.0-109.0) & NS \\
\hline
\end{tabular}

Data are presented as median and interquartile range (in parentheses). The difference between the groups is marked when $p<0.05$ (Mann-Whitney $U$ test), NS - nonsignificant.

Moreover, a statistically significant difference was observed in TAS $(2: 0)$ ratio with a meaningful decrease in group 1. TBARS concentration was elevated 1 day after surgery in both groups, and remained at an elevated level in group 1 , while in group 2 it decreased (Figures 3,4).

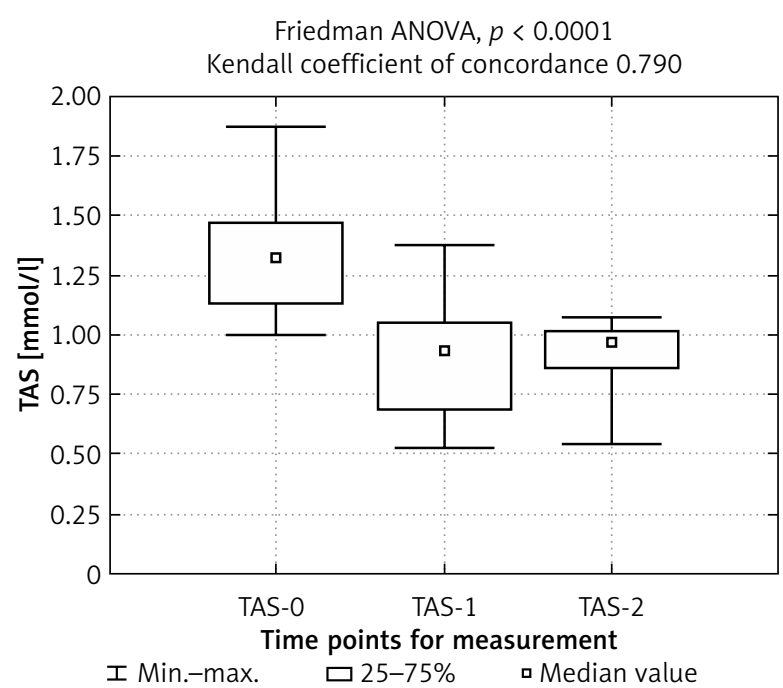

Figure 1 . The kinetics of total antioxidant status (TAS) in group 1
TBARS $(2: 0)$ and TBARS $(2: 1)$ ratios were significantly elevated in group 1 . Finally, higher TAS $(2: 1) /$ TBARS $(2: 1)$ and TAS $(2: 0) / \operatorname{TBARS}(2: 0)$ indexes were obtained in group 2. Detailed preoperative and postoperative data of oxidative stress markers of the two study groups are presented in Table II.

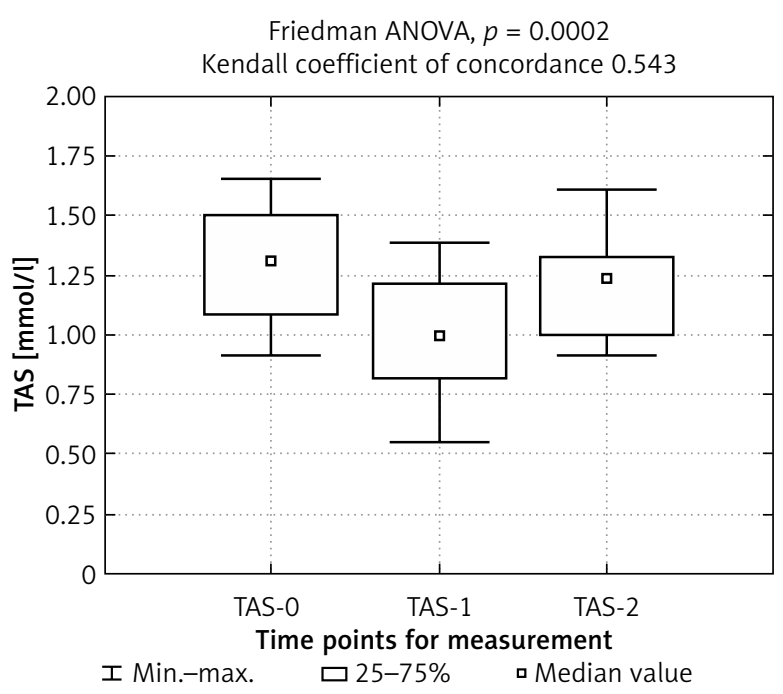

Figure 2. The kinetics of total antioxidant status (TAS) in group 2 


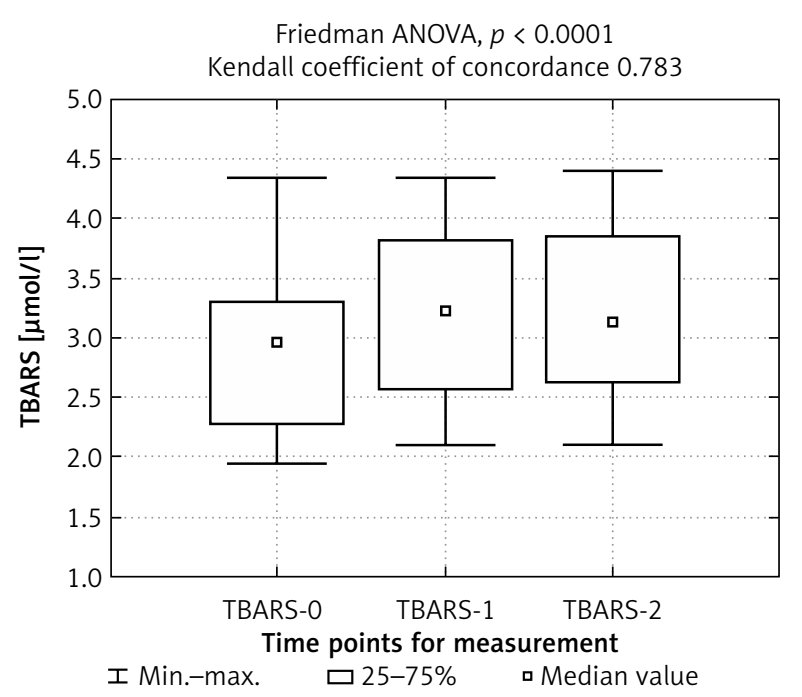

Figure 3. The kinetics of thiobarbituric acid-reactive substances (TBARS) in group 1

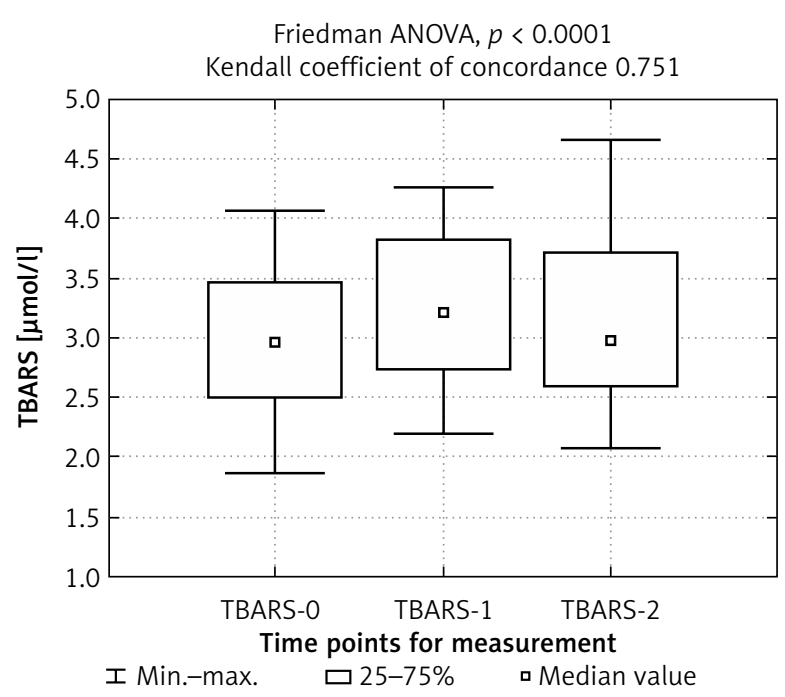

Figure 4. The kinetics of thiobarbituric acid-reactive substances (TBARS) in group 2

Table II. Results

\begin{tabular}{|c|c|c|c|}
\hline Parameter & Group $1(n=17)$ & Group $2(n=17)$ & Significant difference, $p$ \\
\hline Duration of surgery [min] & $50.0(45.0-52.5)$ & $57.5(52.5-65.0)$ & 0.0286 \\
\hline TAS-0 [mmol/l] & $1.31(1.13-1.47)$ & $1.30(1.08-1.50)$ & NS \\
\hline TAS-1 [mmol/l] & $0.93(0.68-1.05)$ & $0.99(0.82-1.22)$ & NS \\
\hline TAS-2 [mmol/ll] & $0.96(0.85-1.01)$ & $1.23(0.99-1.33)$ & 0.0018 \\
\hline TAS $(1: 0)$ ratio & $0.64(0.56-0.78)$ & $0.74(0.68-0.833)$ & NS \\
\hline TAS $(2: 1)$ ratio & $1.05(0.83-1.24)$ & $1.241(1.025-1.45)$ & NS \\
\hline TAS $(2: 0)$ ratio & $0.68(0.62-0.82)$ & $0.96(0.68-0.833)$ & 0.0002 \\
\hline TBARS-O $[\mu \mathrm{mol} / \mathrm{l}]$ & $2.95(2.25-3.30)$ & $2.96(2.48-3.46)$ & NS \\
\hline TBARS-1 [umol/l] & $3.21(2.55-3.82)$ & $3.19(2.72-3.83)$ & NS \\
\hline TBARS-2 [umol/l] & $3.13(2.61-3.84)$ & $2.97(2.57-3.70)$ & NS \\
\hline TBARS $(1: 0)$ ratio & $1.12(1.06-1.20)$ & $1.10(1.05-1.16)$ & NS \\
\hline TBARS $(2: 1)$ ratio & $1.00(0.98-1.05)$ & $0.97(0.92-0.98)$ & 0.0192 \\
\hline TBARS $(2: 0)$ ratio & $1.13(1.04-1.22)$ & $1.03(1.01-1.06)$ & 0.0332 \\
\hline TAS $(1: 0) / \operatorname{TBARS}(1: 0)$ & $0.61(0.50-0.73)$ & $0.72(0.63-0.76)$ & NS \\
\hline $\operatorname{TAS}(2: 1) / \operatorname{TBARS}(2: 1)$ & $1.07(0.85-1.23)$ & $1.30(1.15-1.41)$ & 0.0262 \\
\hline $\operatorname{TAS}(2: 0) / \operatorname{TBARS}(2: 0)$ & $0.63(0.53-0.72)$ & $0.91(0.86-0.98)$ & $<0.0001$ \\
\hline
\end{tabular}

Data are presented as median and interquartile range (in parentheses). The difference between the groups is marked when $p<0.05$ (Mann-Whitney $U$ test), NS - nonsignificant; 0 - before surgery; 1 - 1 day after surgery; 2 - 4 days after surgery.

\section{Discussion}

Laparoscopic surgery techniques were developed to improve treatment outcomes, reduce post-opera- tive complications, accelerate healing and return to normal physical activity as well as minimize cosmetic defects in patients. Any form of trauma, including surgery, is known to result in oxidative stress devel- 
opment. In many clinical trials the severity of metabolic results of surgery-caused injury has been ascertained based on the assessment of oxidative stress/ antioxidant status parameters, e.g. malondialdehyde (MDA), total antioxidant capacity, thiobarbituric acid substances, reduced glutathione, superoxide dismutase (SOD), catalase, glutathione peroxidase (GPX), oxidized LDL autoantibodies (oLAB), neopterin [3]. It is not clear which of these parameters is the best indicator of the oxidative stress response. Of the many indicators of oxidative stress we decided to use TBARS and TAS, which have been used in many trials aimed at assessing tissue trauma after surgery [22-26]. Results of studies comparing open and laparoscopic methods are greatly divergent, with some studies indicating less oxidative stress following laparoscopic surgery [27], others pointing to increased oxidative stress with laparoscopic surgery [8], and still others finding no differences in terms of oxidative stress between open and laparoscopic surgery [28]. Lack of a clear advantage of laparoscopic techniques compared to open techniques may be caused by increased intra-abdominal pressure during pneumoperitoneum and inflation-deflation, which may cause ischemia reperfusion and can subsequently lead to the elevation of oxidative stress level during laparoscopic surgery [29]. Further development of laparoscopic surgery technique focused on decreasing the number of incisions in single incision laparoscopic surgery, which is also used in hernia operations. Available scientific evidence does not demonstrate a clear clinical advantage of single incision laparoscopic surgery used in hernia repair over traditional laparoscopic surgery in terms of length of hospital stay, operative time, pain, recurrence, post-operative complications, issues related to ergonomics, surgical learning curve, and equipment needs [15-18, 30-32]. Our study tries to join in the discussion about advantages of TEP-SI over TEP by revealing the differences in oxidative stress levels between two types of surgery. It seems that if TEP-SI cannot be shown as less invasive than TEP, its only advantage would be slightly better cosmesis, since TEP-SI does not address more vexing problems related to hernia surgery, e.g. postoperative neuropathy, which causes chronic pain in patients who have undergone hernia surgery $[33,34]$. So far, no other authors have compared metabolic responses between TEP and TEP-SI. Less invasive TEP-SI character may eventuate - although it has to be stressed that this is only a conjecture - from the fact that although the sum of lengths of all three incisions performed in TEP $(13 \mathrm{~mm}+6 \mathrm{~mm}+6 \mathrm{~mm})$ was equal to the length of one incision performed in TEP-SI $(25 \mathrm{~mm})$, in TEP-SI only one localized inflammatory response area is present instead of three inflammatory focuses in TEP. The small patient group and using only two oxidative stress factors are suggested to be the limitations of our study. However, this preliminary short-term observation may attract clinicians' attention to the problem of perioperative metabolic stress and the significance of its reduction.

\section{Conclusions}

Our study provides preliminary evidence that oxidative stress levels are lower in patients treated operatively by TEP-SI technique than by TEP. Despite longer duration of surgery, TEP-SI may be considered as a less invasive technique associated with less tissue injury.

\section{Conflict of interest}

The authors declare no conflict of interest.

\section{References}

1. Yiannakopoulou EC, Nikiteas N, Perrea D, Tsigris C. Effect of laparoscopic surgery on oxidative stress response: systematic review. Surg Laparosc Endosc Percutan Techn 2013; 23: 101-8.

2. Bolla G, Tuzzato G. Immunologic postoperative competence after laparoscopy versus laparotomy. Surg Endosc 2003; 17: 1247-50.

3. Arsalani-Zadeh R, Ullah S, Khan S, MacFie J. Oxidative stress in laparoscopic versus open abdominal surgery: a systematic review. J Surg Res 2011; 169: 59-68.

4. Gal D, Lind L, Lovecchio JL, Kohn N. Comparative study of laparoscopy vs. laparotomy for adnexal surgery: efficacy, safety, and cyst rupture. J Gynecol Surg 1995; 11: 153-8.

5. Zullo F, Falbo A, Palomba S. Safety of laparoscopy vs laparotomy in the surgical staging of endometrial cancer: a systematic review and metaanalysis of randomized controlled trials. Am J Obstetr Gynecol 2012; 207: 94-100.

6. Walker JL, Piedmonte MR, Spirtos NM, et al. Laparoscopy compared with laparotomy for comprehensive surgical staging of uterine cancer: Gynecologic Oncology Group Study LAP2. J Clin Oncol 2009; 27: 5331-6.

7. Karuna ST, Thirlby R, Biehl T, et al. Cost-effectiveness of laparoscopy versus laparotomy for initial surgical evaluation and treatment of potentially resectable hepatic colorectal metastases: a decision analysis. J Surg Oncol 2008; 97: 396-403.

8. Bentes de Souza AM, Rogers MS, Wang CC, et al. Comparison of peritoneal oxidative stress during laparoscopy and laparotomy. J Am Assoc Gynecol Laparosc 2003; 10: 65-74. 
9. Rao PP, Rao PP, Bhagwat S. Single-incision laparoscopic surgery - current status and controversies. J Min Acc Surg 2011; 7: 6-16.

10. Hu H, Xu AA. Towards the Holy Grail: what can we do for truly scarless surgery? World J Gastrointest Endosc 2015; 7: 814-8.

11. Schmitt A, Crochet P, Knight S, et al. Single-port laparoscopy vs conventional laparoscopy in benign adnexal diseases: a systematic review and meta-analysis. J Minim Invas Gynecol 2017; 24: 1083-95.

12. Simons MP, Aufenacker T, Bay-Nielsen M, et al. European Hernia Society guidelines on the treatment of inguinal hernia in adult patients. Hernia 2009; 13: 343-403.

13. $\mathrm{Kim} \mathrm{JH}, \mathrm{An} \mathrm{CH}$, Lee YS, et al. Single incision laparoscopic totally extraperitoneal hernioplasty (SIL-TEP): experience of 512 procedures. Hernia 2015; 19: 417-22.

14. Choi BJ, Jeong WJ, Lee IK, Lee SC. Single-port versus conventional three-port laparoscopic totally extraperitoneal inguinal hernia repair: a randomized controlled trial. Hernia 2016; 20: 789-95.

15. Lo CW, Yang SS, Tsai YC, et al. Comparison of laparoendoscopic single-site versus conventional multiple-port laparoscopic herniorrhaphy: a systemic review and meta-analysis. Hernia 2016; 20: 21-32.

16. Cardinali L, Mazzetti CH, Cadenas Febres A, et al. Prospective randomized study comparing single-incisionl aparoscopic versus multi-trocar laparoscopic totally extraperitoneal (TEP) inguinal hernia repair at 2 years. Surg Endosc 2018; 32: 3262-72.

17. Rajapandian S, Bhushan C, Sabnis SC, et al. Single incision multiport versus conventional laparoscopic inguinal hernia repair: a matched comparison. J Minim Access Surg 2018; 14: 44-51.

18. Wakasugi M, Masuzawa T, Tei M, et al. Single-incision totally extraperitoneal inguinal hernia repair: our initial 100 cases and comparison with conventional three-port laparoscopic totally extraperitoneal inguinal hernia repair. Surg Today 2015; 45: 606-10.

19. Chung do Y. The intracorporeal knot tying using rigid instruments in single-incision laparoscopic surgery. Surg Laparosc Endosc Percutan Tech 2013; 23: e90-2.

20. Marcus HJ, Seneci CA, Hughes-Hallett A, et al. Comparative performance in single-port versus multiport minimally invasive surgery, and small versus large operative working spaces: a preclinical randomized crossover trial. Surg Innov 2016; 23: 148-55.

21. Ohkawa H, Ohishi N, Yagi K. Assay for lipid peroxides in animal tissues by thiobarbituric acid reaction. Anal Biochem 1979; 95: 351-8.

22. Koksal H, Kurban S. Total oxidant status, total antioxidant status, and paraoxonase and arylesterase activities during laparoscopic cholecystectomy. Clinics 2010; 65: 285-90.

23. Aktimur R, Gokakin AK, Deveci K, et al. Oxidative stress markers in laparoscopic vs. open appendectomy for acute appendicitis: a double-blind randomized study. J Minim Access Surg 2016; 12: $143-7$

24. Altin FH, Yildirim HA, Tanidir IC, et al. Alterations in antioxidant and oxidant status of children after on-pump surgery for cyanotic and acyanotic congenital heart diseases. Cardiol Young 2018; 28: 325-32

25. Melek FE, Baroncini LAV, Repka JCD, et al. Oxidative stress and inflammatory response increase during coronary artery bypass grafting with extracorporeal circulation. Rev Bras Cir Cardiovasc 2012; 27: 61-5.

26. Starkopf J, Tamme K, Zilmer M, et al. The evidence of oxidative stress in cardiac surgery and septic patients: a comparative study. Clin Chim Acta 1997; 262: 77-88.

27. Polat C, Kahraman A, Yilmaz S, et al. A comparison of the oxidative stress response and antioxidant capacity of open and laparoscopic hernia repairs. J Laparoendosc Adv Surg Techn A 2003; 13: 167-73.

28. McHoney M, Eaton S, Wade A, et al. Inflammatory response in children after laparoscopic vs open Nissen fundoplication: randomized controlled trial. J Pediatr Surg 2005; 40: 908-13.

29. Sammour T, Mittal A, Loveday BP, et al. Systematic review of oxidative stress associated with pneumoperitoneum. Br J Surg 2009; 96: 836-50.

30. Sajid MS, Khawaja AH, Sayegh M, Baig MK. A systematic review comparing single-incision versus multi-incision laparoscopic surgery for inguinal hernia repair with mesh. Int J Surg 2016; 29: 25-35.

31. Fransen SA, Mertens LS, Botden SM, et al. Performance curve of basic skills in single-incision laparoscopy versus conventional laparoscopy: is it really more difficult for the novice? Surg Endosc 2012; 26: 1231-7.

32. Santos BF, Reif TJ, Soper NJ, Hungness ES. Effect of training and instrument type on performance in single-incision laparoscopy: results of a randomized comparison using a surgical simulator. Surg Endosc 2011; 25: 3798-804.

33. Mitura K, Śmietański M, Kozieł S, et al. Factors influencing inguinal hernia symptoms and preoperative evaluation of symptoms by patients: results of a prospective study including 1647 patients. Hernia 2018; 22: 585-91.

34. Mitura K, Garnysz K, Wyrzykowska D, Michałek I. The change in groin pain perception after transabdominal preperitoneal inguinal hernia repair with glue fixation: a prospective trial of a single surgeon's experience. Surg Endosc 2018; 32: 4284-9.

Received: 15.11.2019, accepted: 13.01.2020. 